\title{
V1974 CYG (NOVA CYG 1992) - A POSSIBLE LINK TO SU UMA STARS
}

\author{
A. RETTER, E. M. LEIBOWITZ, E. O. OFEK \\ Tel-Aviv University, Israel
}

V1974 Cyg erupted on 1992 February 19, and was the brightest nova since V1500 Cyg. It soon became an object of massive international multiwavelength observational study.

Some major photometric features of the nova were investigated by DeYoung \& Schmidt (1994), and Semeniuk et al. $(1994,1995)$. They found two periodicities in the nova light curve. While the shorter $0.081263 \mathrm{~d}$ period was present during the 1993 and 1994 observations, the longer $0.0850 \mathrm{~d}$ period was only observed from 1994 July. Semeniuk et al. (1995) interpreted the shorter period as the orbital period of the binary system, and the longer one as caused by the rotation of the white dwarf due to a magnetic field.

We observed V1974 Cyg during 8 nights at the end of 1994. The photometry was carried out with the $1 \mathrm{~m}$ telescope at the Wise Observatory, using the Tektronix $1 \mathrm{~K} \times 1 \mathrm{~K} \mathrm{CCD}$ camera. We observed the star with the standard $B V R I$ filters, concentrating mainly on the $I$ band.

Our results also confirm undoubtedly the presence of two independent periods: $0.08127 \mathrm{~d}$ and $0.08505 \mathrm{~d}$. The persistence of the shorter period over more than one year, and the possible eclipse shaped structure of its folded light curve at the end of 1994, suggest that it is the orbital period of the binary system. The second period is about 4.7 percent longer than the assumed orbital period. The period range and the difference between them are similar to those found in SU UMa stars.

The well known relation between the two periods of SU UMa stars is shown in Fig. 1 (Stolz \& Schoembs 1981, 1984), where the point representing the two periods of V1974 Cyg (asterisk) is also marked. The correspondence is remarkable.

This fact suggests a possible linkage between the two periods of the classical nova V1974 Cyg and the superhump phenomenon in SU UMa stars. Note that the orbital period of the nova falls at the middle of the range of the binary periods of the SU UMa stars. Further indication of the link comes from the fact that the light curve shape of the longer period of 


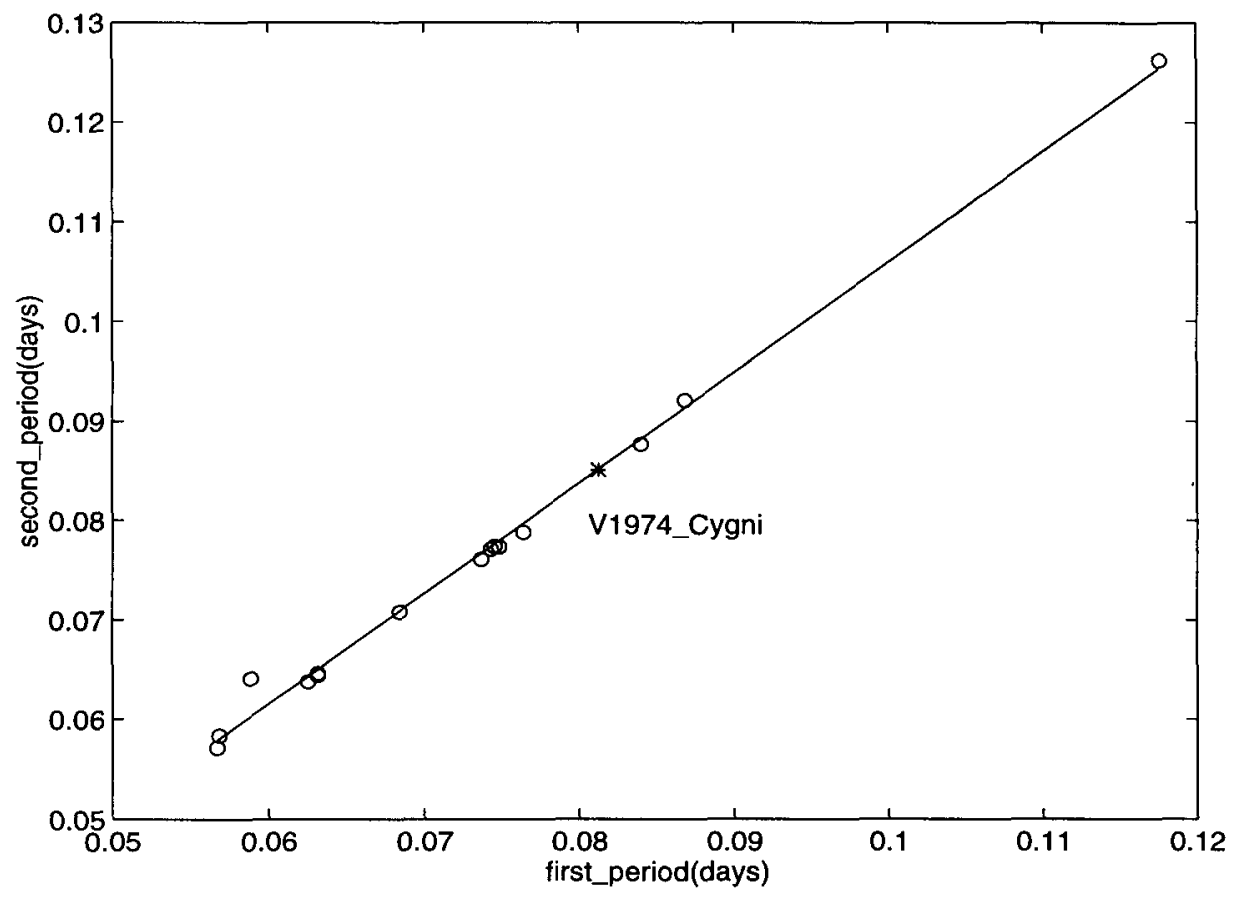

Figure 1. The relation between the orbital and superhump periods in SU UMa stars. The asterisk corresponds to V1974 Cyg's periodicities.

the nova is very much like the shape of the superhump periodicity in a few SU UMa stars (see e.g. la Dous 1993).

The appearance of a new periodicity in the nova power spectrum in the summer of 1994 suggests that an additional light source became dominant. The observational facts compatible with the SU UMa phenomenon suggest, that it is a precessing accretion disk. According to this picture, the nova might experience a long SU UMA like superoutburst a few months after the eruption of the nova.

\section{References}

DeYoung, J.A., Schmidt, R.E., 1994, Ap. J., 431, L47

la Dous, C., 1993, in "Cataclysmic Variables \& Related Objects", ed. Hack M., la Dous, C., Centre National de la Recherce Scientifique, Paris, France, p49

Semeniuk, I., Pych, W., Olech, A., Ruszkowski, M., 1994, Acta Astron., 44, 277

Semeniuk, I., DeYoung, J.A., Pych, W., et al., 1995, Acta Astron., 45, 365

Stolz, B., Schoembs, R., 1981, IBVS 2029

Stolz, B., Schoembs, R., 1984, A\&A, 132, 187 